\title{
Neuromuscular taping application in counter movement jump: biomechanical insight in a group of healthy basketball players
}

\author{
Giuseppe Marcolin (1,2), Alessandro Buriani (1), Andrea Giacomelli (1), David Blow \\ (3), Davide Grigoletto (2), Marco Gesi (4)
}

(1) Motion capture laboratory, Maria Paola Belloni Center for Personalized Medicine, Gruppo Data Medica Padova spa, Padova, Italy; (2) Department of Biomedical Sciences, University of Padova, Italy; (3) Neuro Muscular Taping Institute, Rome, Italy; (4) Department of translational research and new technologies in medicine and surgery, University of Pisa, Italy.

This article is distributed under the terms of the Creative Commons Attribution Noncommercial License (CC BY-NC 4.0) which permits any noncommercial use, distribution, and reproduction in any medium, provided the original author(s) and source are credited.

\begin{abstract}
Kinesiologic elastic tape is widely used for both clinical and sport applications although its efficacy in enhancing agonistic performance is still controversial. Aim of the study was to verify in a group of healthy basketball players whether a neuromuscular taping application (NMT) on ankle and knee joints could affect the kinematic and the kinetic parameters of the jump, either by enhancing or inhibiting the functional performance. Fourteen healthy male basketball players without any ongoing pathologies at upper limbs, lower limbs and trunk volunteered in the study. They randomly performed 2 sets of 5 counter movement jumps (CMJ) with and without application of Kinesiologic tape. The best 3 jumps of each set were considered for the analysis. The Kinematics parameters analyzed were: knees maximal flexion and ankles maximal dorsiflexion during the push off phase, jump height and take off velocity. Vertical ground reaction force and maximal power expressed in the push off phase of the jump were also investigated. The NMT application in both knees and ankles showed no statistically significant differences in the kinematic and kinetic parameters and did not interfere with the CMJ performance. Bilateral NMT application in the group of healthy male basketball players did not change kinematics and kinetics jump parameters, thus suggesting that its routine use should have no negative effect on functional performance. Similarly, the combined application of the tape on both knees and ankles did not affect in either way jump performance.
\end{abstract}

Key Words: neuro muscular taping, vertical jump, biomechanics, performance.

Many injuries commonly reported in basketball, such as ankle sprains and internal knee derangement, have been suggested to be preventable by means of taping, bracing and neuromuscular training. ${ }^{1}$ The effect of taping in reducing ankle injuries has been previously reported. ${ }^{2}$ In particular Garric and Requa ${ }^{3}$ analyzed 2562 basketball player games and reported an injury rate of $14.7 / 1000$ for taped ankles while untaped ankles reveled an injury rate of $32.8 / 1000$. They also found an injury rate of $6.9 / 1000$ in the 288 player games where elastic tape was employed. Interestingly it has been shown that a certain amount of vigorous exercise reduced the mechanical properties of the non-elastic tape in supporting the ankle. ${ }^{2}$ The effectiveness of non-elastic tape in diminishing the injury rate, together with the reduction of its mechanical properties after vigorous exercise, are suggestive of an addictive proprioceptive stimulation of normal neuromuscular mechanisms for protection against injurious movement. ${ }^{2}$ Considering the more dynamic characteristics of elastic tape, it seems to be reasonable to think that it could be equally or even more effective than non-elastic tape. ${ }^{2}$ However consensus on this issue has not been reached yet and some authors challenge the effectiveness of taping in injury prevention. ${ }^{4,5}$

Kinesiologic tape is a water resistant elastic band that can stretch up to $120-140 \%$ of its original length and can adhere to the skin for 3-5 days without the necessity of substitution, and with a very low level of skin irritation. ${ }^{6}$ According to the tape use guidelines, ${ }^{6}$ the tension of the tape on the skin generates convolutions which elevate the epidermis, reducing the pressure on the mechanoreceptors situated under that portion of skin. With the same mechanism this technique can 
improve blood and lymphatic circulation, thus contributing to pain intensity reduction, joints realignment and improvement of muscle activity. ${ }^{6}$ Kinesiologic tapes are widely used by physical and manual therapists in clinical practice despite a lack of consistency in scientific evidences. Indeed, even if some researchers have reported a positive effect on short term pain reductiom, ${ }^{7-9}$ and on trunk range of motion, ${ }^{10}$ others have shown no substantial benefit on muscle tone and strength, ${ }^{11}$ foot posture $^{12}$ and circulation. ${ }^{13}$ Taking together these results, Kinesiologic tape efficacy appears to be still controversial in clinical practice, ${ }^{14}$ and further research is needed. Likewise, studies on Kinesiologic tape applications in sports, have given contrasting results, showing height in jump performance unchanged $^{15}$ or decreased, ${ }^{16}$ push-off force increased, ${ }^{16}$ dynamic postural control increased, ${ }^{15}$ no effect on muscle strength ${ }^{17}$ and muscle endurance. ${ }^{13}$ Taken together the results on the effectiveness of Kinesiologic tape in sport performance applications indicates that further investigation is required. ${ }^{5,18}$

However these previous studies investigated only single biomechanical parameters such as jump height and vertical force, or scores for balance assessment ${ }^{15}$ and level of proprioception ${ }^{19}$ and the kinesiologic tape was applied considering only a single joint (knee or ankle).

Neuromuscular taping (NMT) application is a relative new technique, where elastic kinesiologic tape is used to create the conditions for passive stretching, with eccentric techniques, with enhancement of flexibility and coordination and increasing of the range of movement in patients suffering of lack of muscle coordination. ${ }^{20-22}$ As reported in two case studies, this application seems to be promising in clinic to improve gait strategy in patients with joint hyper mobility syndrome/Ehlers-Danlos syndrome hyper mobility type $^{20}$ and to improve upper limb movement in patients with cerebral palsy. ${ }^{22}$

On the basis of these previous observations, we focused on sport application of kinesiologic tape, and in particular on studying the biomechanics of the jump, in order to investigate whether in this context NMT application could affect kinematic and kinetic parameters of knees and ankles, with enhancing or inhibitory effects on the functional performance. The neuromuscular taping technique was also tested for its potential combined effect when applied on more than one joint. Our hypothesis was that the elasticity of the tape did not affect joints range of motion, while its application on more than one joint could increase jump performance. The measurements were conducted in a group of healthy basketball players performing counter movement jumps with and without the application of the elastic kinesiologic tape on both knees and ankles.

\section{Material and Methods}

Fourteen male basketball players were enrolled for the present study (mean age 24 ; mean weight $81 \pm 10 \mathrm{~kg}$; mean height $184 \pm 8 \mathrm{~cm}$ ). At the time of the tests participants did not present any pathology in the upper limbs, lower limbs and trunk. An informed consent, including an explanation of all procedures was read approved and signed by each participant before undergoing the experimental procedures. The study was reviewed and approved by the local Public Ethics Committee (Comitato Etico per la Sperimentazione Clinica della Provincia di Padova).

Each participant performed the test in a one day session. After a standardized warm up exercise consisting of 5 minutes running on a treadmill and 2 stretching exercises for tights and calves, participants were asked to perform 2 sets of 5 counter movement jumps (CMJ) with hands on the hips. Resting time between jumps was 1 minute while the interval between the two sets of jumps was 10 minutes. The order of the first set of jumps with or without the tape was randomly assigned. When participants performed the first set of jumps without tape this was applied during the 10 minutes resting time between sets of jumps.

Kinematic data were recorded with the a SMART-D optoelectronic system (BTS Bioengineering, Milan, Italy), which consists of eight infrared video cameras.
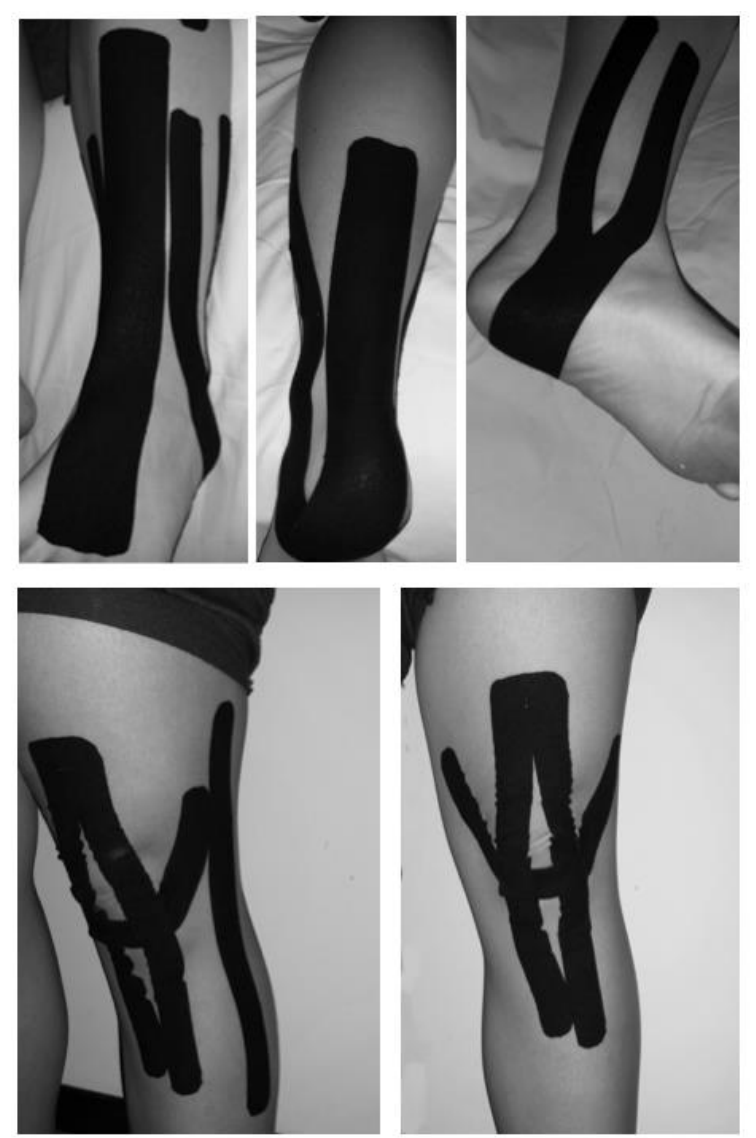

Fig 1. Neuromuscular taping application adopted for knee and ankle taping in the group of basketball players. 

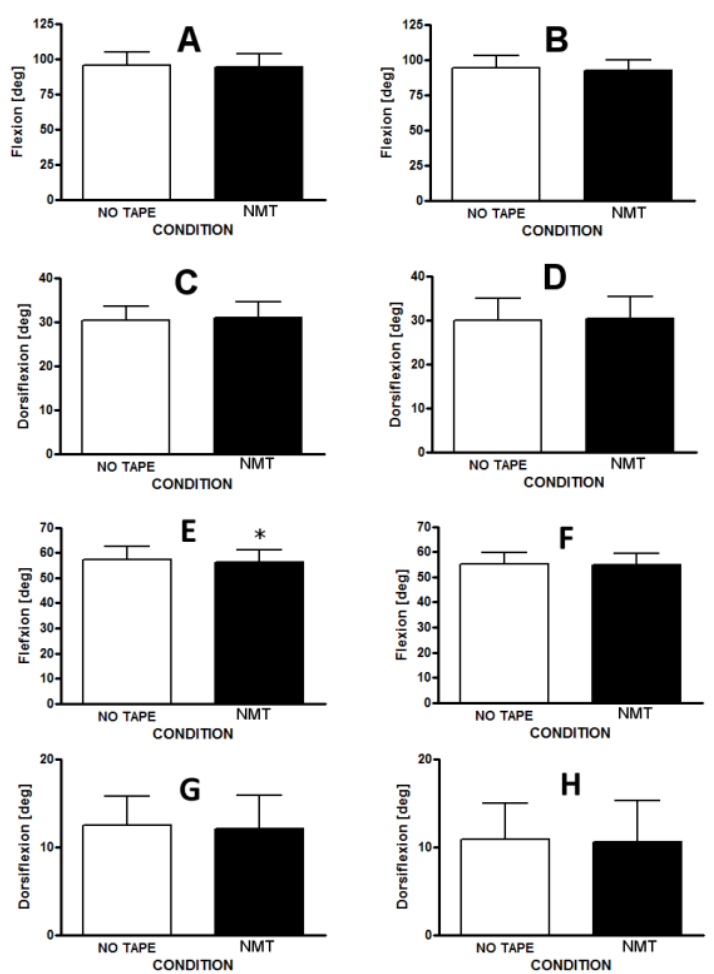

Fig 2. Maximal knee flexion and ankle dorsiflexion before the beginning of the push off phase. Values of left (A and C) and right side (B and D). Knee flexion and ankle dorsiflexion correspondent to the maximal jump power during the push off phase. Values of left (E and $\mathrm{G}$ ) and right side $(\mathrm{F}$ and $\mathrm{H})$.

All data are expressed as means and standard deviations $(* p<.05)$

Kinetic data were synchronously recorded on by means of two Kistler platforms (Kistler Group, Winterthur, Switzerland) embedded in the floor. The Kinematic data were sampled at sampling rate was $200 \mathrm{~Hz}$, and kinetic data were collected at $800 \mathrm{~Hz}$. Passive reflective markers were bilaterally placed respectively on the great trochanter, on the lateral knee condyle, on the fibula head and on the lateral malleolus. Two markers were stuck on the shoes, in correspondence of the heel and of the fifth metatarsal head. Finally a marker was placed on the midpoint between the posterior superior iliac spines. kinesiologic tape was placed on knees and ankles of all the participants by the same certified manual therapist. The application of the tape regarded both ankles and knees (Figure 1) and followed the indications proposed by Blow. 21 In particular, with knee flexed at 90 degrees, one tape $(25 \mathrm{~cm}$ length and $2.5 \mathrm{~cm}$ width) was applied on the patella covering the inferior margin for $1 \mathrm{~cm}$ and following its medial and lateral margin. The level of tension of the tape corresponded to the $25 \%$ of the maximal tension allowed. A second tape with $Y$ shape $(30 \mathrm{~cm}$ length and
$5 \mathrm{~cm}$ width) was fixed proximally on the centre of the thigh with the two tails following the lateral and medial margins of the patella and distally on the tibial tuberosity. During its application, the knee was flexed at 110 degrees and no tension was applied. Two other tapes $(25 \mathrm{~cm}$ length and $2.5 \mathrm{~cm}$ width) were placed respectively along the medial and lateral knee ligaments with level of tension of $25 \%$ and subject in a standing posture.

A tape with $\mathrm{X}$ shape was stuck on each ankle starting from a neutral position $\left(90^{\circ}\right.$ angle between foot and shank). Two tails surrounded the medial malleolus and two tails the lateral malleolus. Their length was approximately $20 \mathrm{~cm}$ and their width $2.5 \mathrm{~cm}$. The level of tension was $50 \%$. A second tape of $5 \mathrm{~cm}$ width was applied anterior from the fingers up to half the length of the tibia with level of tension of $25 \%$. Finally a tape of 5 $\mathrm{cm}$ width was fixed under the heel and applied with a $50 \%$ level of tension along the achilles tendon.

The best 3 jumps in term of height each participant performed both with and without kinesiologic tape were selected for data analysis and mean values were obtained. Kinematics and kinetics were assessed through a customized tool in SMART Analyzer (BTS Bioengineering, Milan, Italy). In particular kinematics parameters were: knees maximal flexion and ankles maximal dorsiflexion, jump height and take off velocity. Kinetics parameters analyzed were: vertical ground reaction force and power of the jump. The knee and ankle angles corresponding at to the maximal power during the push off phase were also calculated.

Wilcoxon signed rank test was carried out to compare jumps performed with kinesiologic tape and jumps performed without the tape. Significant level was set for $\mathrm{p}<0.05$. Data analysis was performed using the software package GraphPad Prism version 4.00 for Windows (GraphPad Software, San Diego California, USA).

\section{Results}

From a kinematic point of view the application of the tape yielded no significant changes in maximal right and left knee flexion, as well as in maximal right and left ankle dorsiflexion (Figure 2). Knee and ankle angles at the maximal power during the push off phase showed no significant differences in the two conditions tested (Figure 2), with the exception of the left knee ( $p=$ 0.0134; effect size: 0.21 ). No statistically significant differences were detected in jump height, take off velocity, vertical force during the push off phase and maximal jump power (Table 1).

\section{Discussion}

When analyzing the application of supports (different kinds of tape application or braces) to the joints of athletes to prevent injuries, one important aspect has to be considered: they should not modify the joint range of motion (ROM), thus influencing negatively their overall performance. This insures that athletes can maintain the 


\section{Neuromuscular taping application in counter movement jump}

Eur J Transl Myol 27 (2): 130-135

Table 1. Biomechanical parameters recorded in the two experimental conditions.

NO TAPE CONDITION

JUMP HEIGHT $(\mathrm{cm})$

TAKE OFF VELOCITY $(\mathrm{m} / \mathrm{sec})$

VERTICAL GRF MAX (BW ratio)

MAX POWER (W/BW)
$49.84 \pm 8.451$

$2.518 \pm 0.331$

$2.496 \pm 0.267$

$48.97 \pm 8.559$
NMT CONDITION

$49.82 \pm 7.963$

$2.524 \pm 0.348$

$2.504 \pm 0.264$

$49.13 \pm 7.714$

Values are expressed as mean and standard deviation. Kinetic data are referred to the push off phase of the jump.

effective technical execution of their sport discipline. The result that there was no significant differences in knee and ankle angles at the maximal power during the push off phase indicates that there is no negative effect on healthy athletes who have obtained a high level of technical jump execution. Cordova and colleagues $^{23}$ reported a lace-up style brace effect on sprint speed with an impairment of speed of approximately $1 \%$, although they concluded the meta-analysis stating that the benefit of ankle support for injury prevention overcame the possibility of small impairment of performance. Metcalfe and colleagues ${ }^{24}$ showed a reduction of ankle/subtalar ROM, together with a decrease of jump and agility performance when using ankle prophylactics.

In basketball players the initial phase of the jump shot can be compared to a counter movement jump with ball on hands. Then, after the take off, the player has to focus on the shooting technique, trying to leave the ball after reaching the maximum jump height. The NMT application we adopted in the present study, considering both ankles and knees, could be considered more invasive than other single applications. Therefore the hypothesis that our combined application could affect the technical execution of the jump was considered. Kinematics and kinetics data showed no statistically significant differences when comparing the two experimental conditions for all the parameters, except the angle of the left knee at the reach of the maximum power during the push off phase. However the difference detected of 1.12 degrees can reasonably be considered trivial from a technical execution point of view. Therefore our hypothesis that NMT application did not affect joint ROM was confirmed by kinematic results. We can thus state that this aspect of jump functional performance remains unaltered when NMT is employed.

The present study also investigated the short-term efficacy of NMT application on jump performance. Previous results both in healthy ${ }^{15,25,26}$ and injured athletes $^{27}$ referred to single joint applications of kinesiologic elastic tape had shown no substantial effects. Thus we investigated a bilateral application on both knees and ankles, hypothesizing an amplified effect to enhance jump performance. However our results showed no added effect on jump height, take off velocity, vertical force and maximal power during the push off phase in the healthy basketball players enrolled. Collectively, despite bilateral application on two joints, our data resulted similar to those of previous investigations on elastic tape and jump performance. ${ }^{15,26,28}$ Therefore our hypothesis of a positive combined effect of NMT when applied on more than one joint was not confirmed by the results of our study. As reported by Moisés de Hoyo and colleagues, ${ }^{26}$ one explanation could be that afferent stimuli generated by kinesiologic tape may have not been strong enough to modulate muscle performance of the healthy athletes, leaving the performance unchanged.

The results of our study line up with others where the effect of kinesiologic elastic tape on muscle strength was investigated. Csapo and colleagues ${ }^{15}$ reported in their meta-analysis how kinesiologic tape had no or negligible effects on muscle strength, thus concluding that knee extensor and flexor, together with ankle plantarflexor, cannot be improved by kinesiologic tape application in young male and female healthy subjects. Our study showed that bilateral NMT application in a group of healthy male basketball players did not affect kinematics and kinetics of the jump. NMT application was not able to increase jump performance, thus aligning with previous results in the literature not supporting the use of these tapes to increase jump performance. Taken together these observations suggest that further investigations on the application of NMT could focus on injury rate reduction during basketball training and competitions, as well as on its effect on injury treatment. In our study only healthy athletes without any physical impairment were included. Thus it is not possible to predict if the mechanism of action of the tape could be more effective on injured athletes or athletes recovering from injury. The principal objective of Neuromuscular Taping is its implied action during rehabilitation from sports trauma or from surgery. The present study analyzed the single vertical jump capacity but further study is needed to take into account the shooting precision during the vertical jump together with the NMT application. Moreover NMT application should be evaluated in others specific basketball skills where jumps are involved as blocking, dunking and 
rebounding. We also studied a specific group of male basketball players, and thus our results might not necessarily apply to female or to athletes of other disciplines.

\section{List of acronyms}

NMT - neuromuscular taping application

CMJ - counter movement jumps

ROM - range of motion

\section{Author's contributions}

GM, AB, AG, Design of the work; GM, AG, DG, Data acquisition and analysis; GM, AB, DB, MG, Interpretation of the data for the work; GM, AB, DG, Draft of the work; DB, MG, Revision for important intellectual content; GM, AB, AG, DB, DG, MG, Final approval of the work.

\section{Acknowledgments}

None external financial support was used for the present study. The authors thank all the voluntary athletes who participated to the study.

\section{Conflict of Interest}

Neuro Muscular Taping NMT ${ }^{\circledR}$ was used for the study and is a register product. Although no external financial support was used for the present study, there is a potential duality of interest for one of the authors, David Blow, who is the founder and president of the Neuro Muscular Taping (NMT) Institute in Rome where NMT was developed.

\section{Corresponding Author}

Buriani Alessandro, Via Zanchi, 89. 35133 Padova, Italy. E-mail: alessandro.buriani@gmail.com

\section{E-mails of coAuthors}

Giuseppe Marcolin: giuseppe.marcolin@unipd.it Andrea Giacomelli: andrea.giacomelli@hotmail.com David Blow: david.blow@nmtinstitute.org Davide Grigoletto: davide.grigoletto@unipd.it Marco Gesi: marco.gesi@med.unipi.it

\section{References}

1. Dick R, Hertel J, Agel J et al. Descriptive epidemiology of collegiate men's basketball injuries: national collegiate athletic association injury surveillance system, 1988-1989 through 2003-2004. J Athl Train 2007;42:194-201.

2. Firer P. Effectiveness of taping for the prevention of ankle ligament sprains. $\mathrm{Br} \mathrm{J}$ Sports Med 1990;24:47-50.

3. Garrick JG, Requa RK. Role of external support in the prevention of ankle sprains. Med Sci Sports 1973;5:200-3.

4. Mostafavifar M, Wertz J, Borchers J. A systematic review of the effectiveness of kinesio taping for musculoskeletal injury. Phys Sportsmed. 2012;40:33-40.

5. Williams S, Whatman C, Hume PA et al. Kinesio taping in treatment and prevention of sports injuries: a meta-analysis of the evidence for its effectiveness. Sports Med 2012; 42:153-164.

6. Kase K, Wallis J, Kase T. Clinical Therapeutic Applications of the kinesio Taping Method. Tokio, Japan: Keni-Kai Co., Ltd.; 2003.

7. Djordjevic OC, Vukicevic D, Katunac L et al. Mobilization with movement and kinesio taping compared with a supervised exercise program for painful shoulder: results of a clinical trial. J Manipulative Physiol Ther 2012;35:454-463.

8. Kalron A, Bar-Sela S. A systematic review of the effectiveness of Kinesio Taping®_ Fact or fashion? Eur J Phys Rehabil Med 2013;49:699709.

9. Taylor RL, O’Brien L, Brown T. A scoping review of the use of elastic therapeutic tape for neck or upper extremity conditions. J Hand Ther 2014;27:235-245.

10. Yoshida A, Kahanov L. The effect of kinesio taping on lower trunk range of motions. Res Sports Med 2007;15:103-112.

11. Gomez-Soriano J, Abian-Vicen J, Aparicio-Garcia $\mathrm{C}$ et al. The effects of Kinesio taping on muscle tone in healthy subjects: a double-blind, placebocontrolled crossover trial. Man Ther 2014;19:131136.

12. Luque-Suarez A, Gijon-Noqueron G, Baron-Lopez FJ et al. Effects of kinesiotaping on foot posture in participants with pronated foot: a quasirandomised, double-blind study. Physiotherapy 2014;100:36-40.

13. Stedge HL, Kroskie RM, Docherty CL. Kinesio taping and the circulation and endurance ratio of the gastrocnemius muscle. J Athl Train 2012; 47:635-642.

14. Parreira P do C, Costa Lda C, Hespanhol Junior LC et al. Current evidence does not support the use of Kinesio Taping in clinical practice: a systematic review. J Physiother 2014;60:31-39.

15. Nakajima MA, Baldridge C. The effect of Kinesio ${ }^{\circledR}$ tape on vertical jump and dynamic postural control. Int $\mathrm{J}$ Sports PhysTher 2013;8:393-406.

16. Huang CY, Hsieh TH, Lu SC et al. Effect of the Kinesio tape to muscle activity and vertical jump performance in healthy inactive people. Biomed Eng Online 2011;10:70.

17. Csapo R, Alegre LM. Effects of Kinesio ${ }^{\circledR}$ taping on skeletal muscle strength- A meta-analysis of current evidence. J Sci Med Sport 2015; 18:450456.

18. Drouin JL, McAlpine CT, Primak KA et al. The effect of kinesiotape on athletic-based performance 


\section{Neuromuscular taping application in counter movement jump}

Eur J Transl Myol 27 (2): 130-135

outcomes in healthy, active individuals: a literature synthesis. J Can Chiropr Assoc 2013;57:356-365.

19. Halseth T, McChesney JW, Debeliso M et al. The effect of kinesio ${ }^{\mathrm{TM}}$ taping on proprioception at the ankle. J Sports Sci Med 2004;3:1-7.

20. Camerota F, Galli M, Cimolin V et al. The effects of neuromuscular taping on gait walking strategy in a patient with joint hypermobility syndrome/Ehlers-Danlos syndrome hypermobility type. Ther Adv Musculoskelet Dis 2015;7:3-10.

21. Blow D. NeuroMuscular Taping: From Theory to Practice. Milano: Edi-ermes. 2012.

22. Camerota F, Galli M, Cimolin V et al. Neuromuscular taping for the upper limb in Cerebral Palsy: A case study in a patient with hemiplegia. Dev Neurorehabil 2014;17:384-387.

23. Cordova ML, Scott BD, Ingersoll CD et al. Effects of ankle support on lower-extremity functional performance: a meta-analysis. Med Sci Sports Exerc 2005;37:635-641.

24. Metcalfe RC, Schlabach GA, Looney MA et al. A comparison of moleskin tape, linen tape, lace-up brace on joint restriction and movement performance. J Athl Train 1997;32:136-140.

25. Merino-Marban R, Mayorga-Vega D, FernandezRodriguez E. Effect of kinesio tape application on calf pain and ankle range of motion in duathletes. $\mathbf{J}$ Hum Kinet 2013; 37:129-135.

26. De Hoyo M, Alvarez-Mesa A, Sanudo B et al. Immediate effect of kinesio taping on muscle response in young elite soccer players. J Sport Rheabil 2013;22:53-58.

27. Bicici S, Karatas N, Baltaci G. Effect of athletic taping and kinesiotaping on measurements of functional performance in basketball players with chronic inversion ankle sprains. Int $\mathrm{J}$ Sports PhysTher 2012;7:154-166.

28. Schiffer T, Mollinger A, Sperlich B et al. Kinesio taping and jump performance in elite female track and field athletes and jump performance in elite female track and field athletes. J Sport Rehabil 2015;24:47-50. 